\title{
First disruption studies and simulations in view of the development of the DEMO Physics Basis
}

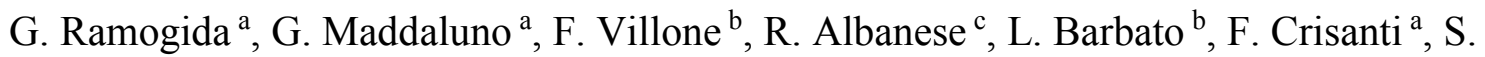

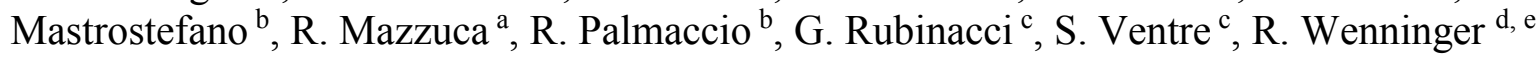 \\ ${ }^{a}$ ENEA for EUROfusion, via E. Fermi 45, 00044 Frascati (Roma), Italy \\ ${ }^{b}$ University of Cassino Consorzio CREATE, Cassino, Italy \\ ${ }^{c}$ University Federico II Consorzio CREATE, Naples, Italy \\ ${ }^{d} I P P$, Garching, Germany \\ ${ }^{e}$ EFDA, Garching, Germany
}

\begin{abstract}
In the development of the DEMO Physics Basis an important role is played by the prediction of the plasma disruption features and by the evaluation of the Electro-Magnetic (EM) and thermal loads associated with these events. Indeed, the kind and number of foreseen plasma disruptions drive the development of the DEMO operation scenarios and the design of vessel and in-vessel components.

To characterize a plausible macroscopic plasma dynamics during these events, we will carry out an extrapolation from present-day machines of the main parameters characterizing the disruptions: thermal and current quench time, evolution of plasma current, $\beta$ and li, safety factor limits, halo current fraction and width, radiated heat fraction. In particular, we will focus on extrapolations for the thermal and current quench characteristic times, due to their importance for the subsequent simulations aimed at the evaluation of the EM and thermal loads. The different options for DEMO design will be taken into account and the possible range of variation of the parameters will be estimated.

The 2D axysimmetric MAXFEA and the 3D CarMa0NL codes will be used to evaluate the effects of the induced currents and the EM loads during a disruptive event and to analyze the various design options obtained by the PROCESS code. The results of these simulations, modeled as worst expected events, will be used as input for the system level analysis and design of the vessel and relevant in-vessel components. First simulations with CarMa0NL code including 3D structures are here presented to show the significant effects due to the large access ports.
\end{abstract}

Keywords: DEMO; plasma disruptions; VDE; MAXFEA; CarMa0NL.

\section{Introduction}

The simulation of reliable plasma disruptions represent a key issue in the design of DEMO and of any large, high performance tokamak, since the huge electromagnetic, mechanical and thermal loads affecting the machine structures during these events, necessarily leads to significant design and operational constraints. It is therefore essential to avoid both under-estimation and over-estimation of their possible consequences that could result in serious damages of the involved structures or in an unbearable costs increase.

In the frame of the EuroFusion PMI Work Package, activities started to simulate the plasma evolution and the induced currents and loads during the worst (from the machine integrity point of view) off-normal events in DEMO. As input for these simulations, it is necessary to estimate reasonable values for the features representing the plasma evolution in these events. The ElectroMagnetic (EM) and thermal loads resulting from these plasma simulations will be used for a variety of purposes, among which: the design and system level analysis of the plasma chamber, access ports and related supports; the design of divertor, blanket, first wall and other in-vessel components; the assessment of plasma control and mitigation systems.

\section{Features of the plasma disruptions expected in DEMO}

A plasma disruption occurs when the plasma suddenly lose most of its thermal energy (thermal quench) and its current drop rapidly to zero (current quench). These events can take place before or after a vertical displacement of the plasma and may involves also significant poloidal current flowing in the passive structure near to the plasma, due to the variation of toroidal flux and to the occurrence of halo currents.

At least three kinds of plasma disruptions need to be simulated in DEMO to provide the EM and thermal loads required for the design: Major Disruption (MD), Downward Vertical Displacement Event (D-VDE) and Upward VDE (U-VDE). In a VDE large vertical forces are exerted mainly on the Vacuum Vessel (VV) due to the vertical asymmetry of the event, then this event will be used to obtain the maximum vertical loads that the VV must withstand. The rather large upper vertical ports foreseen in DEMO are particularly concerning from the vertical stability point of view, due to the reduced stabilizing effect in this region, then the U-VDE simulation is a priority for the design of the VV. 
A first set of input features required to carry out the disruption simulations were estimated with the same approach used in ITER Physics Basis [1, 2], i.e. extrapolating them from current machines data. Actually, even if the features obtained with these extrapolation result in disruption events rather conservative, there is no justification strong enough to exclude the occurrence of these events in the DEMO operation. For this reason, these features have to be chosen for the disruption simulations carried out to evaluate the engineering design loads. The estimation was made comparing the reference DEMO 1 design with three design options at different aspect ratio, summarized in Table 1.

Table 1. Some features for the DEMO options at different aspect ratios, computed by PROCESS code.

\begin{tabular}{lllll}
\hline & $\begin{array}{l}\text { DEMO1 } \\
\mathrm{A}=4\end{array}$ & $\begin{array}{l}\text { DEMO } \\
\mathrm{A}=3.6\end{array}$ & $\begin{array}{l}\text { DEMO } \\
\mathrm{A}=3.1\end{array}$ & $\begin{array}{l}\text { DEMO } \\
\mathrm{A}=2.6\end{array}$ \\
\hline $\mathrm{A}$ & 4.0 & 3.6 & 3.1 & 2.6 \\
\hline $\mathrm{R}_{0}[\mathrm{~m}]$ & 9.0 & 9.13 & 8.77 & 8.91 \\
\hline $\mathrm{a}[\mathrm{m}]$ & 2.25 & 2.536 & 2.828 & 3.427 \\
\hline $\mathrm{k}_{95}$ & 1.66 & 1.62 & 1.55 & 1.51 \\
\hline$\delta_{95}$ & 0.33 & 0.333 & 0.333 & 0.333 \\
\hline $\mathrm{S}^{*}\left[\mathrm{~m}^{2}\right]$ & 26.3 & 59.7 & 39.0 & 30.5 \\
\hline $\mathrm{S}_{\mathrm{p}}\left[\mathrm{m}^{2}\right]$ & 1127 & 1212 & 1314 & 1651 \\
\hline $\mathrm{V}_{\mathrm{p}}\left[\mathrm{m}^{3}\right]$ & 1527 & 1819 & 2214 & 3404 \\
\hline $\mathrm{I}_{\mathrm{p}}[\mathrm{MA}]$ & 16.0 & 17.7 & 20.3 & 24.2 \\
\hline $\mathrm{B}_{\text {tor }}\left(\mathrm{R}_{0}\right)[\mathrm{T}]$ & 7.06 & 7.04 & 5.80 & 4.25 \\
\hline $\mathrm{q}_{95}$ & 3.0 & 3.0 & 3.0 & 3.0 \\
\hline$\beta_{\mathrm{N}, \text { tot }}[\%]$ & 2.44 & 2.453 & 2.585 & 2.799 \\
\hline
\end{tabular}

\subsection{Thermal Quench duration}

The Thermal Quench (TQ) represents the internal redistribution and loss to Plasma Facing (PF) surfaces of the thermal energy $W_{\text {th }}$, starting immediately before the onset of disruption. It consists in a slow phase $\left(\tau_{1-2}\right)$, due to large scale locked modes, and in a fast redistribution of toroidal current $\left(\tau_{2}\right)$, accompanied with a flattening of the profile $\left(l_{i}\right.$ decrement) and a positive current spike (around one tenth of the plasma current), due to the flux conservation.

In MD most of the thermal energy (up to 90\%) is radiated during the slow phase, instead in hot plasma VDE and high $\beta_{\mathrm{N}}$ internal pressure-gradient triggered disruptions (ITB), less than $10 \%$ of energy is dissipated during the slow TQ phase and the remainder is lost in the fast TQ when the plasma touch the PF components (mainly divertor in D-VDE).

The experimental data from existing machines suggest a scaling of the thermal quench duration by the minor radius $[2,3]$ : extrapolation of typical TQ times to DEMO yields values for the fast TQ $\left(\tau_{2}\right)$ ranging from $0.8 \mathrm{~ms}$ for the reference pulsed DEMO 1 up to $1.1 \mathrm{~ms}$ for the low aspect ratio design option; in a similar way the duration of the pre-disruptive slow TQ phase $\left(_{\tau 1-2}\right)$ varies from 21 to $32 \mathrm{~ms}$. Table 2 shows the minimum TQ characteristic times for JET with the relevant extrapolations to ITER $[2,4]$ and various DEMO options with different aspect ratio.
The actual duration of the fast TQ $\left(\tau_{2}\right)$ is uncertain, due to the finite time response of the Soft X Rays (SXR) diagnostics used in the thermal loss data. Some fast ECE measurements in JET [2] during prompt ITB disruptions show values short as one tenth of the related SXR data. Extrapolation of these measurements to DEMO yields values for the fast TQ $\left(\tau_{2}\right)$ duration around $0.1 \mathrm{~ms}$ : anyway the time-scale of transport of thermal energy to the PF surfaces could prevent the potentially damaging effect of such very fast TQ. Moreover, from the EM point of view, the simulations are not affected by the shortening of this duration that is anyway essentially instantaneous, given the time constants of the EM effects.

Table 2. Extrapolation of the thermal quench duration time for JET, ITER and DEMO options.

\begin{tabular}{lllllll}
\hline & & \multicolumn{5}{c}{ DEMODEMO DEMO DEMO } \\
& JET & ITER & $1 \mathrm{~A}=4$ & $\mathrm{~A}=3.6$ & $\mathrm{~A}=3.1$ & $\mathrm{~A}=2.6$ \\
\hline $\mathrm{a}[\mathrm{m}]$ & 0.95 & 2.00 & 2.25 & 2.536 & 2.828 & 3.427 \\
\hline $\operatorname{tau}(1-2)[\mathrm{ms}]$ & 9.00 & 18.95 & 21.32 & 24.03 & 26.79 & 32.47 \\
\hline $\operatorname{tau}(2)[\mathrm{ms}]$ & 0.32 & 0.67 & 0.76 & 0.85 & 0.95 & 1.15 \\
\hline $\operatorname{tau}(2-\mathrm{ITB})[\mathrm{ms}]$ & 0.05 & 0.11 & 0.12 & 0.13 & 0.15 & 0.18 \\
\hline
\end{tabular}

\subsection{Current Quench duration}

The Current Quench (CQ) occurs after the plasma has lost most of its thermal energy, when its resistivity has increased. This current decay induces toroidal eddy currents in the nearby conducting structure (net toroidal current in the VV and local saddle currents on the blanket modules). The entity of these currents and of the associated EM loads strongly depends on their vertical asymmetry (due to the plasma motion) and on the current decay rate and is the main responsible of the severity of the disruption events from an EM point of view.

Some helical currents may flow in the structures contacting the halo region that lies beyond the last closed plasma surface. The time evolution and the motion of the plasma during the CQ are partly balanced by the induced eddy and the halo currents. The halo currents reconnecting through vessel and in-vessel conducting structures produces in-vessel poloidal halo currents through these structures and then relevant local and global EM loads on them.

The range of values for the CQ rates were estimated using the database of disruption in existing tokamak [2], corrected excluding circular tokamaks and including updated JT60U and JET data [5], that allows to set a lower limit, independent of elongation and toroidal current density, for the area-normalized global current decay time $\Delta \mathrm{t}_{100} / \mathrm{S}^{*}=1.8 \mathrm{~ms} / \mathrm{m}^{2}$, i. e. the time the plasma current employs to go to zero, normalized by the plasma cross-section area in elliptic approximation, $\mathrm{S}^{*}=$ $\pi \mathrm{ka}^{2}$.

The CQ duration for an extremely fast disruption is also been evaluated, as in ITER, using the extrapolation factor deduced from the previous full database including circular machines [1]: in this case the lower limit for the 
area-normalized global current decay time is $\Delta \mathrm{t}_{100} / \mathrm{S} *=$ $1.33 \mathrm{~ms} / \mathrm{m}^{2}$. This event should be classified as extremely unlikely event for safety purposes.

The $\Delta \mathrm{t}_{100} / \mathrm{S}$ * values have been estimated supposing a linear decay of the plasma current during the CQ. The analysis of JT-60U disruption data suggests actually a Sshaped CQ waveform, with an intermediate current decay phase that is best fitted by an exponential function when the area-normalized CQ time is small and by a linear decay otherwise [6]. The approximation of an exponential decay with a linear extrapolation results in an approximately 2.2 underestimation factor for the peak rate of current decay. Anyway the difference in the CQ effects between linear and exponential waveform appears only when analyzing structures with time constants shorter than the CQ decay time and does not affect the estimation of global magnetic pressures and forces in large structures

The application of these extrapolations to DEMO yields a lower limit for $\Delta \mathrm{t}_{100}$ ranging from $47 \mathrm{~ms}$ for the reference pulsed DEMO 1 up to to $107 \mathrm{~ms}$ for the low aspect ratio design option; in a similar way the duration of the exponential CQ time constant $\tau_{\mathrm{CQ}}$ varies from 21 to $48 \mathrm{~ms}$ and should be used in the simulations carried out to evaluate local forces on in-vessel components. The minimum CQ duration for the extremely fast disruption, using the IPB-1999 scaling, instead varies between 35 and $80 \mathrm{~ms}$. Table 3 show the extrapolated CQ time for JET, ITER and various DEMO options with different aspect ratio. The CQ durations measured in JET during $\mathrm{C}$ wall operations are consistent with the minimum value showed in the table [7] while the durations observed with the Be ITER Like Wall are longer as discussed in the following paragraph. This involves a possible overestimation of the maximum CQ decay rate in ITER.

Table 3. Extrapolation of the minimum current quench duration time and constant for JET, ITER and DEMO options.

\begin{tabular}{lllllll}
\hline & \multicolumn{5}{c}{ DEMO1 DEMO DEMO DEMO } \\
& JET & ITER & $\mathrm{A}=4$ & $\mathrm{~A}=2.6$ & $\mathrm{~A}=3.1$ & $\mathrm{~A}=3.6$ \\
\hline $\mathrm{a}[\mathrm{m}]$ & 0.95 & 2 & 2.25 & 3.427 & 2.828 & 2.536 \\
\hline $\mathrm{k}(95)$ & 1.6 & 1.7 & 1.66 & 1.62 & 1.55 & 1.51 \\
\hline $\mathrm{S}^{*}\left[\mathrm{~m}^{2}\right]$ & 4.54 & 20 & 26.3 & 59.7 & 39.0 & 30.5 \\
\hline $\begin{array}{l}\text { CQ_extreme } \\
\text { duration [ms }]\end{array}$ & 6.03 & 26.60 & 35.01 & 79.40 & 51.86 & 40.60 \\
\hline $\begin{array}{l}\text { CQ fast } \\
\text { duration [ms }]\end{array}$ & 8.17 & 36.00 & 47.38 & 107.46 & 70.19 & 54.95 \\
\hline $\begin{array}{l}\text { CQ time (exp) } \\
{[\mathrm{ms}]}\end{array}$ & 3.63 & 16.00 & 21.06 & 47.76 & 31.20 & 24.42 \\
\hline
\end{tabular}

\subsection{Effects due to the plasma facing materials}

The recent JET experiments with ITER Like Wall (ILW) [8] pointed out that the lack of a good radiating impurity like carbon results in a fraction of radiated power at disruption much smaller than before. In turn this results in longer current quenches, larger heat loads caused by conduction of magnetic energy to Plasma Facing Components (PFC) and longer [8] halo currents. The forces produced by these long lasting halo currents, applied on the vessel with $\mathrm{Be}$ wall, produce larger reaction forces on the vessel supports than with the $\mathrm{C}$ wall, due to its longer characteristic times. Temperatures above the melting limit of beryllium have been observed on upper first wall structures during deliberate VDE, at relatively low plasma current and thermal energy content. Indeed this is due to the dissipation of the magnetic energy by conduction on a limited area for the ILW rather than by radiation everywhere uniformly as in the C-wall.

This led to the present mandatory use in JET of Massive Gas Injection (MGI) to mitigate disruptions for currents $\mathrm{I}_{\mathrm{p}}>=2.5 \mathrm{MA}$. In JET the low amount of released or mobilized particles released during the thermal quench, together with the low radiation efficiency of beryllium, are responsible for the strong reduction in radiation.

In ITER, with the same plasma facing material pattern (Be-wall and W-divertor), but with larger thermal and magnetic energy, the foreseen heat load on the divertor overcomes by far the maximum allowed value of merit figure for $\mathrm{W}$ melting $\left(50 \mathrm{MJ} \mathrm{m}^{2} \mathrm{~s}^{-0.5}\right.$ ), even with $50 \%$ degradation of pre-disruptive thermal energy. Even more critical the situation for the Be wall (Be melt limit: $25 \mathrm{MJ} \mathrm{m} \mathrm{m}^{-0.5}$ ). Disruption mitigation appears to be mandatory.

In ASDEX-Upgrade the difference between disruption behavior going from a full $\mathrm{C}$ to a full $\mathrm{W}$ machine was not clearly evident. This was due to the smooth (8 years) transition in plasma facing material with significant residual $\mathrm{C}$ in the machine and to the prescribed disruptions mitigation by Massive Gas Injection that has been operating for many years. New data analysis [9] has shown that with all W PFCs and boronised walls, suppressing the residual $\mathrm{C}$ influence, a larger fraction of the total energy is deposited in the divertor and the $\mathrm{CQ}$ lasts longer, in most cases, with respect to the all $\mathrm{C}$ device. Anyway short $\mathrm{CQ}$ durations were occasionally observed also with $\mathrm{W}$ wall.

For DEMO, with the first wall made of tungsten, besides the divertor, the possibility that the influx to the core of tungsten eroded during the TQ from the first wall may result in a large radiated power should be evaluated.

\section{Disruption simulations}

A comprehensive activity of plasma disruptions simulations for DEMO has been started using the 2D plasma equilibrium solver MAXFEA and the 3D code CarMa0NL. First models with MAXFEA were affected by some inaccuracies due to the rather toroidally large access ports put in DEMO which make the axisymmetric meshes inefficient from the vertical stability point of view.

\subsection{CarMa0NL models}

A 3D model of the vessel was created by using the CarMa0NL code [10], able to describe the nonlinear evolution of free-boundary axisymmetric plasmas in 
presence of $3 \mathrm{D}$ conducting structures surrounding the plasma.

The CarMa0NL code considers a coupling surface which is used to decouple the EM interaction among plasma and structures. In the plasma region, inside the coupling surface, a 2D triangular finite elements mesh is considered to model the plasma evolution by solving the Grad-Shafranov equation. Instead in the conductors region, outside the coupling surface, a $3 \mathrm{D}$ hexahedral volumetric mesh of the conductive structures in a sector is considered. The vacuum do not need to be meshed, since an integral formulation is used for the eddy currents equations. Figure 1 shows the two meshes used for the plasma region (on the left) and the conductive vacuum vessel and blanket (on the right).

We stress that the results of such a 3D code can be used to derive the parameters of suitable simpler equivalent axisymmetric models, to be used e.g. for controller design. Anyway, it should be noted that a 2D model with a modification only of the toroidal resistivity of the vessel (e.g. to take into account the presence of the ports), may be too simplistic. Indeed, doing so, we would not take into account the 3D inductive effects, which affect a number of key control parameters, like the stability margin of plasma configurations.
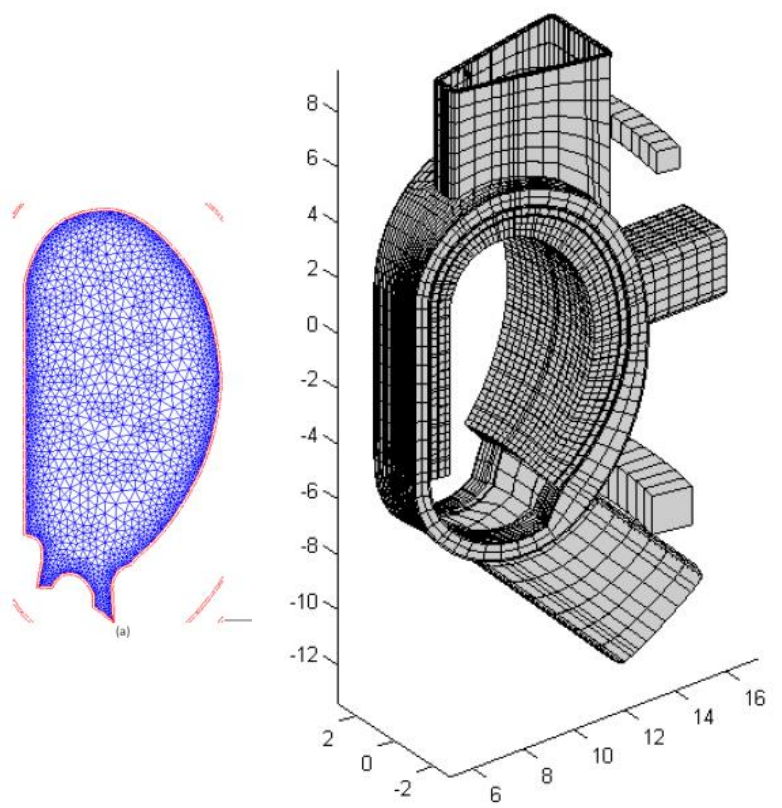

Fig. 1. CarMa0NL mesh for the 2D axisymmetric plasma region (on the left) and the $3 \mathrm{D}$ conductor region (on the right).

The upward plasma vertical displacement in CarMa0NL is simulated driving a voltage kick in P2-P5 coils, connected in antiseries so as to provide a mainly radial magnetic field. The polarity is chosen so as to move the plasma upwards.

The plasma is then left free to evolve with frozen plasma current and current density profiles until it hits the first wall. After the plasma-wall contact, the Thermal
Quench is modeled with a poloidal beta drop to $10 \%$ of its nominal value in $1 \mathrm{~ms}$. For all the following time steps, the poloidal beta is kept fixed to this very low value.

Just after the TQ, the plasma current is supposed to increase of $10 \%$ of its nominal value in $1 \mathrm{~ms}$; simultaneously the internal inductance decreases of a similar amount, by imposing a flattening of the current density profile. Then the Current Quench starts, during which the plasma current is imposed linearly decreasing to zero in $47 \mathrm{~ms}$, keeping the same flattened current density profile.

\subsection{Upward VDE simulation results}

The simulation of the plasma evolution during an upward VDE excited by an external voltage kick, has been carried out with the CarMa0NL mesh including full $3 \mathrm{D}$ details of the main conducting structures (vacuum vessel, main access ports and blanket-equivalent modules).

The main plasma parameters have been computed, together with significant data regarding the structures and equivalent filamentary currents: these filaments, providing the same magnetic field as the plasma outside the coupling surface, are available for further detailed electromagnetic analyses of specific components. Figure 2 shows the time evolution of the main plasma features: plasma current $\mathrm{I}_{\mathrm{p}}$, toroidal flux $\Phi_{\text {tor }}$, plasma center $\mathrm{R}_{\mathrm{c}}$ and $Z_{c}, \beta_{\text {pol }}$ and $1_{i}$, safety factor $q_{95}$. We recall that plasma current, internal inductance and poloidal beta are inputs of the analysis, while the other quantities are results of the computation.
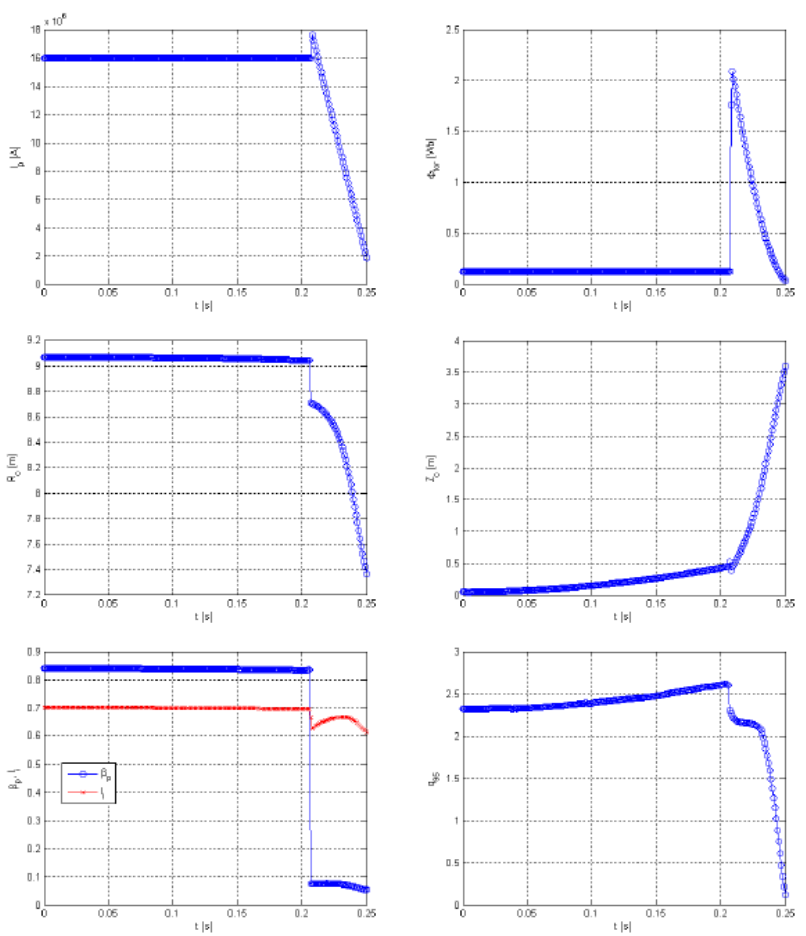

Fig. 2. Time evolution of the main computed plasma features: from left to right and from top to bottom, $I_{p}$ $[\mathrm{A}], \Phi_{\mathrm{tor}}[\mathrm{Wb}], \mathrm{R}_{\mathrm{c}}[\mathrm{m}], \mathrm{Z}_{\mathrm{c}}[\mathrm{m}], \beta_{\mathrm{pol}}$ and $\mathrm{l}_{\mathrm{i}}$, q95. 
In the simulation the plasma hit the First Wall at $\mathrm{t}=$ 206 ms: at this time the TQ first and the CQ later were imposed. The plasma current fell below 1.5 MA at $\mathrm{t}=$ $251 \mathrm{~ms}$ : later, no more convergence was achieved, due to absence of halo currents, so the plasma current was forced to zero. Halo current have not been included in the analysis, since no extrapolation to DEMO of expected halo widths and total halo currents have been made yet. This important point is left to future activity.

Due to the relatively fast CQ time scale, as compared to typical electromagnetic time constants, the maximum induced toroidal current is very close to the predisruption total plasma current, as shown in Figure 3 on the left. The maximum poloidal current shows a large peak during the TQ, when the toroidal flux increases appreciably, due to high pre-disruption value of poloidal beta, and goes back to zero during the CQ, as shown in Figure 3 on the right.
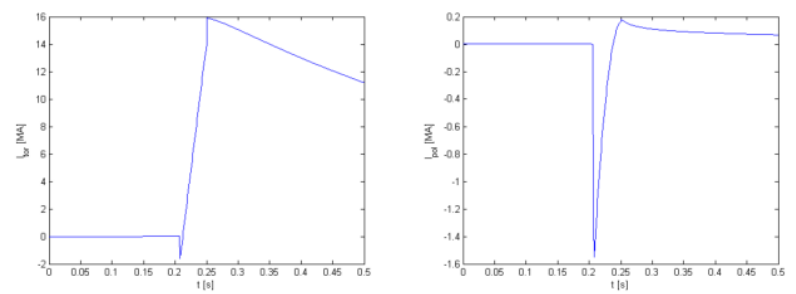

Fig. 3. Total toroidal (on the left) and poloidal (on the right) currents induced in the conductive structures during the U-VDE.

The induced eddy current pattern is initially concentrated on the outer shell, close to the PF coils which are actively driving the plasma kick, later it shifts to the expected up-down anti-symmetric $m=1$ pattern during the vertical displacement and finally approach to a $m=0$ pattern during the CQ. The evolution of plasma and eddy current pattern during the U-VDE is shown in Figure 4.
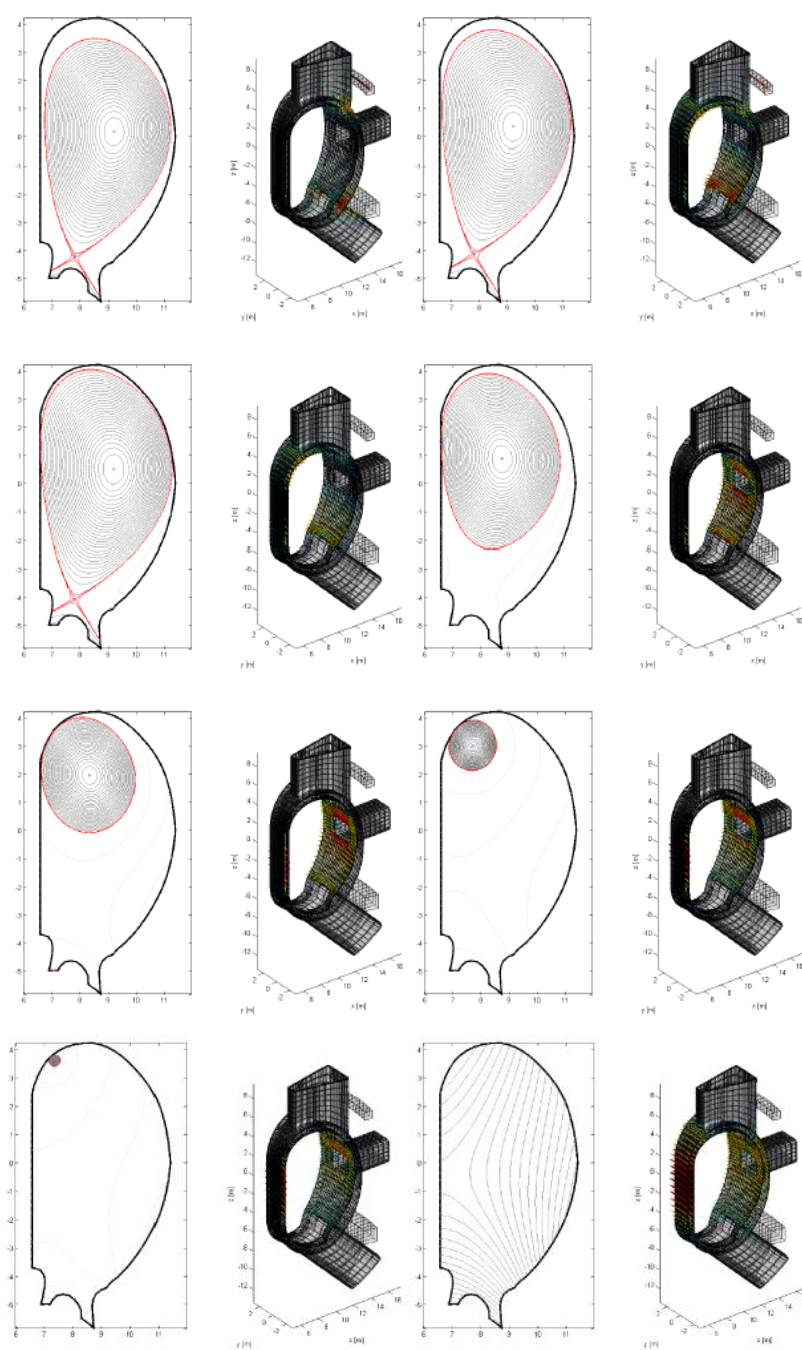

Fig. 4. Snapshots of the plasma configurations and corresponding current density patterns in the $3 \mathrm{D}$ vessel during the U-VDE at $\mathrm{t}=50,150,200,220,235,245$, 251 and $400 \mathrm{~ms}$.

\section{Conclusions}

The design of DEMO requires the prediction of plausible plasma disruption features and the evaluation of the EM and thermal loads associated with these events. The activities described here are the extrapolation of the Thermal and Current Quench times and the first simulation by the 3D code CarMa0NL of a VDE including the effects due to the large access ports that make difficult the use of axisymmetric codes as MAXFEA. A discussion on the effect of the Plasma Facing materials in the disruptions is also presented and will be deepened in the future when more experimental data from machines with metallic materials will be available.

\section{Acknowledgments}

This work has been carried out within the framework of the EUROfusion Consortium and has received funding from the Euratom research and training 
programme 2014-2018 under grant agreement No 633053. The views and opinions expressed herein do not necessarily reflect those of the European Commission.

This work has been partly supported by Italian MIUR under PRIN grant No 2010SPS9B3.

\section{References}

[1] ITER Physics Expert Group on Disruptions, Plasma Control and MHD and ITER Physics Basis Editors, Nucl. Fusion 39 (1999) 2251-2390.

[2] T. C. Hender et al., Nucl. Fusion 47 (2007) S128-S202.

[3] F. C. Schuller, Plasma Phys. Control. Fusion 37 (1995) A135-Al62.

[4] V. Riccardo et al., Nucl. Fusion 45 (2005) 1427-1438.

[5] M. Sugihara et al., Analysis of disruption scenarios and their possible mitigation in ITER, Proceedings 20th IAEA Fusion Energy Conference, Villamoura, Portugal (2004) IT/P3-29.

[6] M. Sugihara et al., J. Plasma and Fusion Research 79 (2003) 706.

[7] V. Riccardo et al., Plasma Phys. Control. Fusion 47 (2005) 117.

[8] M. Lehnen et al., Nucl. Fusion 53 (2013) 093007.

[9] R. Neu et al., "Experiences with Tungsten Plasma Facing Components in Asdex Upgrade and JET", IEEE Trans. on Plasma Science, 42 (2013) 552 - 562.

[10] F. Villone et al., Plasma Phys. Control. Fusion 55 (2013) 095008 . 